\title{
Role of GTP Hydrolysis in Microtubule Dynamics: Information from a Slowly Hydrolyzable Analogue, GMPCPP
}

\author{
A.A. Hyman, ${ }^{*}$ S. Salser, + D.N. Drechsel, + N. Unwin, $\neq$ \\ and T.J. Mitchison*
}

\author{
*Department of Pharmacology and †Biochemistry and Biophysics, University of California \\ San Francisco, San Francisco, California 94143; and łLab of Molecular Biology, \\ Medical Research Council, Cambridge, United Kingdom
}

Submitted June 19, 1992; Accepted August 3, 1992

\begin{abstract}
The role of GTP hydrolysis in microtubule dynamics has been reinvestigated using an analogue of GTP, guanylyl- $(\alpha, \beta)$-methylene-diphosphonate (GMPCPP). This analogue binds to the tubulin exchangeable nucleotide binding site (E-site) with an affinity four to eightfold lower than GTP and promotes the polymerization of normal microtubules. The polymerization rate of microtubules with GMPCPP-tubulin is very similar to that of GTPtubulin. However, in contrast to microtubules polymerized with GTP, GMPCPP-microtubules do not depolymerize rapidly after isothermal dilution. The depolymerization rate of GMPCPP-microtubules is $0.1 \mathrm{~s}^{-1}$ compared with $500 \mathrm{~s}^{-1}$ for GDP-microtubules. GMPCPP also completely suppresses dynamic instability. Contrary to previous work, we find that the $\beta-\gamma$ bond of GMPCPP is hydrolyzed extremely slowly after incorporation into the microtubule lattice, with a rate constant of $4 \times 10^{-7} \mathrm{~s}^{-1}$. Because GMPCPP hydrolysis is negligible over the course of a polymerization experiment, it can be used to test the role of hydrolysis in microtubule dynamics. Our results provide strong new evidence for the idea that GTP hydrolysis by tubulin is not required for normal polymerization but is essential for depolymerization and thus for dynamic instability. Because GMPCPP strongly promotes spontaneous nucleation of microtubules, we propose that GTP hydrolysis by tubulin also plays the important biological role of inhibiting spontaneous microtubule nucleation.
\end{abstract}

\section{INTRODUCTION}

Microtubules are a noncovalent helical polymer of the protein tubulin, a heterodimer of similar $\alpha$ and $\beta$ subunits (Kirschner, 1978; Purich and Kristoffersen, 1984). In living cells, microtubules exist in an unusual dynamic equilibrium with tubulin subunits, termed dynamic instability. Individual microtubules alternate stochastically between prolonged periods of polymerization and depolymerization, leading to rapid exchange between tubulin subunits and microtubule polymer (reviewed in Gelfand and Bershadsky, 1991). Similar behavior is exhibited by microtubules polymerized from pure tubulin in vitro (Mitchison and Kirschner, 1984; Walker et al., 1988). From these observations, dynamic instability has been proposed to play a key role in generating the asymmetric microtubule arrays the cell uses for its mi- crotubule dependent functions (Kirschner and Mitchison, 1986).

Dynamic instability is a highly nonequilibrium behavior and thus requires an energy source. For pure tubulin in vitro, this energy comes from GTP hydrolysis by tubulin. Each tubulin dimer has two GTP binding sites, an exchangeable (E) site on the $\beta$ subunit and a nonexchangeable site on the $\alpha$ subunit that is always filled with GTP. Under conditions thought to be physiological, the tubulin E-site must be occupied by GTP to polymerize into microtubules. During or after polymerization, the E-site GTP is hydrolyzed to GDP that is trapped in the microtubule (reviewed in Kirschner, 1978; Purich and Kristoffersen, 1984; Carlier, 1989). The most widely quoted model to explain the mechanism of dynamic instability is the GTP cap model (Mitchison and Kirschner, 1984; Chen and Hill, 1985; Kirschner 
and Mitchison, 1986; Carlier, 1989; Caplow, 1992). This model proposes that although the bulk of the tubulin polymer has hydrolyzed GTP to GDP in the E-site, there exists a cap of subunits at the end of a growing microtubules that have not hydrolyzed their GTP and therefore stabilize the microtubule against depolymerization. Loss of the cap leads to microtubule depolymerization. The thermodynamic argument in the GTP cap model is that the full free energy of GTP hydrolysis is only released when the microtubule depolymerizes and that tubulin has evolved GTP hydrolysis to destabilize the polymer.

The evidence for the GTP cap model comes from three types of experiment: kinetic analysis of tubulin polymerization, measurement of GTP hydrolysis, and experiments with nonhydrolyzable GTP analogues. Kinetic analysis has been the most important (Mitchison and Kirschner, 1984; Walker et al., 1988) but cannot provide direct evidence for biochemical mechanism. Measurement of GTP hydrolysis rates has been controversial. At the time the GTP cap model was proposed, a large kinetic lag was thought to exist between tubulin polymerization and GTP hydrolysis (Carlier and Pantaloni, 1981). More recent measurements have shown that GTP hydrolysis is in fact closely coupled to polymerization, and it has been difficult to determine whether a GTP cap really exists (reviewed in Caplow, 1992). Thus, GTP analogue experiments are an important test of the model. The GTP cap model predicts that a nonhydrolyzable GTP analogue should 1) promote normal polymerization, 2) block depolymerization, and 3 ) block dynamic instability as a consequence of 2).

Analysis of microtubule polymerization promoted by the non-hydrolyzable analogues guanalyl-imidodiphosphate (GMPPNP) and guanalyl $(\beta$-y-methylene)diphosphonate (GMPPCP) in fact preceded the GTP cap model and provided some of the evidence for it. These analogues support polymerization of normal microtubules in the absence of GTP hydrolysis, confirming prediction 1) above (Arai and Kaziro, 1976; Weisenberg and Deery, 1976; Penningroth and Kirschner, 1977, 1978; Mejillano et al., 1990; Seckler et al., 1990). However, polymerization rates were not measured at the level of individual microtubules in these studies. Thus the effect of the analogues on the on-rate during polymerization is not known, though it is clear that polymerization is slower than with GTP (Seckler et al., 1990). Prediction 2) has not been tested definitely in experiments with GMPPNP and GMPPCP. Microtubules polymerized with these analogues were found to be more stable to depolymerization by cold and calcium ions than GDP microtubules (Arai and Kaziro, 1976; Weisenberg and Deery, 1976), leading to the idea that nucleotide hydrolysis is important for microtubule depolymerization. However, these conditions are not relevant to the spontaneous isothermal depolymerization of microtubules that occurs during dynamic instability. GMPPNP and GMPPCP in fact suffer some problems as tools to analyze the role of GTP hydrolysis in microtubule dynamics. Their affinity for the tubulin E-site is much lower than GTP (Karr and Purich, 1978; O'Brien and Erickson, 1989), requiring that the endogenous E-site nucleotide be exhaustively stripped before polymerization experiments (Mejillano et al., 1990; Seckler et al., 1990). As well as introducing technical problems, this large difference in binding affinity between GTP and analogue complicates any thermodynamic analysis of the effects of hydrolysis.

In this article, we analyze microtubule polymerization dynamics in the presence of GMPCPP. This analogue was shown previously to bind well to the tubulin E-site and to promote vigorous polymerization. However, it was reported to be hydrolyzed during polymerization reactions (Sandoval et al., 1977, 1978) and has thus not been considered a nonhydrolyzable analogue. guanylyl$(\alpha, \beta)$-methylene-diphosphonate (GMPCPP) stimulates a large amount of nucleation and growth of unusually stable microtubules when injected in vivo (Wehland and Sandoval, 1983), effects that resemble those of the microtubule stabilizing drug taxol (DeBrabander et al., 1981). We suspected GMPCPP might cause these effects because it was not hydrolyzed and thus stabilized the microtubules against depolymerization. In this article, we reinvestigated the hydrolysis question and found that GMPCPP is hydrolyzed by bovine brain microtubules so slowly as to be considered a nonhydrolyzable analogue, despite its normal $\beta-\gamma$ phosphoanhydride bond. We also measured the key rate-constants that control microtubule dynamics in the presence of GMPCPP. Our results provide strong support for the GTP-cap model for dynamic instability, as well as suggesting an important role for GTP hydrolysis in suppressing spontaneous nucleation.

\section{MATERIALS AND METHODS}

\section{Synthesis of GMPCPP}

GMPCPP is no longer commercially available, so we developed a synthesis protocol by adapting the dicyclohexylcarbodiimide procedure for the synthesis of adenylyl $\alpha-\beta$-methylene diphosphonate (AMPCPP) (Myers et al., 1965). Because of the technical usefulness of the analogue, we will describe our procedure in detail. To make GMPCP $8 \mathrm{mmol}$ isopropylidene-guanosine (Sigma, St. Louis, MO), $32 \mathrm{mmol}$ methylene diphosphonic acid (Lancaster Synthesis, Lancashire, England), $63 \mathrm{mmol}$ di-cyclohexylcarbodiimide (Aldrich Chemical, Milwaukee, WI) and $50 \mathrm{ml}$ tri-butylamine (Aldrich) were dissolved in $400 \mathrm{ml}$ pyridine (Aldrich anhydrous grade). The reaction was stirred at $60^{\circ} \mathrm{C}$ for $6 \mathrm{~h}$, during which time dicyclohexylurea separates as a fine precipitate. Solvent was removed in vacuo, the mixture was resuspended in water, filtered, and the cake was washed with water. The combined filtrate was adjusted to $\mathrm{pH} 7$ if necessary and extracted four times with di-ethyl ether. The aqueous phase was dried down and resuspended in water twice and then dried down and resuspended in $200 \mathrm{ml} 10 \%$ acetic acid and incubated $100^{\circ} \mathrm{C}$ for $2 \mathrm{~h}$ to hydrolyze the isopropylidine group. The mixture was then dried down and resuspended in water four times. The final solution $(100 \mathrm{ml})$ was adjusted to $\mathrm{pH} 8.6$ with $\mathrm{NaOH}$. The main ultraviolet (UV) absorbing species in the mix was now GMPCP by thin-layer chromatography (TLC) (see below), which was purified by ion exchange chromatography. One-half the product was loaded onto a $950-\mathrm{ml}$ column of DEAE-ToyoPearl (Supelco, Bellefonte, PA) equilibrated with $0.05 \mathrm{M}$ tri-ethylammonium bicarbonate (TEAB), $\mathrm{pH}$ 7.5. The column was 
eluted with a linear gradient $0.05-0.2$ with a M TEAB, 61 total volume. The major OD255 absorbing peak was pooled, and the solvent was removed by rotary evaporation. The solid was resuspended in methanol plus $1 \mathrm{ml}$ tri-butyl amine, dried down, and resuspended in methanol twice more. GMPCP was precipitated as its sodium salt from the product in a small volume of methanol by addition of several volumes of $1 \mathrm{M} \mathrm{NaI}$ in acetone. The white powder was collected by centrifugation, washed twice with acetone, and dried in vacuo.

To convert GMPCP to GMPCPP, we made up a solution containing $5 \mathrm{mM}$ GMPCP, $50 \mathrm{mM}$ tris(hydroxymethyl)aminomethane (Tris) $\mathrm{Cl}$, $10 \mathrm{mM} \beta$-mercaptoethanol, $10 \mathrm{mM} \mathrm{MgCl}, 20 \mathrm{mM}$ acetylphosphate (Sigma), $20 \mu \mathrm{g} / \mathrm{ml}$ Escherichia Coli acetate kinase (Sigma). The mixture was adjusted to $\mathrm{pH} 7.5$ with acetic acid before adding the enzyme. It was incubated at $25^{\circ} \mathrm{C}$ for $48 \mathrm{~h}$, readjusting the $\mathrm{pH}$ with $\mathrm{NaOH}$ several times. The final conversion to GMPCPP was $\sim 75 \%$ by TLC. To purify the GMPCPP, a reaction equivalent to one-fourth the original GMPCP synthesis was loaded directly onto the DEAE column above, equilibrated with $0.1 \mathrm{M} \mathrm{TEAB}$, and developed with a gradient of $0.1-0.4$ $M$ TEAB. The major peak was pooled, and the GMPCPP was obtained as a dry sodium salt as above. It was stored at $-80^{\circ} \mathrm{C}$ as a desiccated powder or made up in water as a $0.1 \mathrm{M}$ stock, assuming $\epsilon_{255}=13500$, and stored frozen for months without sign of decomposition. The total yield was $\sim 20 \%$ based on guanosine, and GMPCPP was the only UV absorbing spot in the final product by TLC. Its identity was confirmed by TLC comigration with the last commercial batch available (ICN, Irvine, CA), ready hydrolysis to GMPCP by alkaline phosphatase, and ready reconversion of the dephosphorylated product to GMPCPP by nucleoside diphosphate kinase + ATP.

To prepare $\gamma^{32} \mathrm{P}-\mathrm{GMPCPP}$, we made $50 \mu \mathrm{l}$ of a solution containing $60 \mu \mathrm{M}$ GMPCP, $1 \mu \mathrm{Ci} \backslash \lambda \gamma^{32} \mathrm{P}$-ATP $(3000 \mathrm{Ci} / \mathrm{mmol}), 50 \mathrm{mM} \mathrm{KCl}$, $20 \mathrm{mM}$ TrisCl, $5 \mathrm{mM} \mathrm{MgCl}$, $10 \mathrm{mM} \beta$-mercaptoethanol, $\mathrm{pH} 7.5$, containing $20 \mu \mathrm{m} / \mathrm{ml}$ of NDP-kinase at $25^{\circ} \mathrm{C}$. After $4 \mathrm{~h}$ to allow equilibration of the label, the labeled GMPCPP was purified by TLC (see below). The product area was determined by autoradiography, eluted with several washes of $1 \mathrm{M}$ TEAB, concentrated by lyophilization, buffer exchanged by gel filtration on Sephadex G-10 in BRB80 (80 mM Kpipes, $1 \mathrm{mM} \mathrm{MgCl}, 1 \mathrm{mM}$ EGTA, $\mathrm{pH} 6.8$ ), and stored at $-80^{\circ} \mathrm{C}$. Recently, we have obtained similar results by simply freeing the NDP-kinase reaction mixture of protein by spinning it through a 10-kDa cutoff membrane (Millipore, Bedford, MA). This gives a final $\gamma^{32}$ P-GMPCPP preparation containing a small amount of cold GMPCP and a tiny amount of $\gamma^{32} \mathrm{P}$-ATP, but neither of these interfere with hydrolysis experiments such as those shown in Figures 5 and 6.

\section{TLC}

Polyethylenimine (PEI) cellulose plates (Alltech, Deerfield, IL) were run in $1.4 \mathrm{M} \mathrm{LiCl}$. Nucleotides were visualized by $\mathrm{UV}$ absorption or autoradiography. $R_{f}$ values were GMPCP 0.50 , GMPCPP 0.38 , GTP 0.2 , GDP 0.35, ATP 0.29, Pi 0.8. For analysis of nucleotides bound to tubulin, the protein was denatured by addition of formamide to $50 \% \mathrm{vol} / \mathrm{vol}$ before spotting. This prevented streaking on the plate.

\section{Tubulin Polymerization}

Calf brain tubulin was purified by temperature-dependent polymerization and phosphocellulose chromatography and then polymerized, sedimented through glycerol cushions, and stored frozen in aliquots as described (Hyman et al., 1991). This preparation contains only the nucleotides that cosediment with the polymer. Tubulin concentration was determined on protein that had been pelleted as microtubules and resuspended without extra nucleotide, using OD277, assuming $\epsilon=80000 \mathrm{M}^{-1} \mathrm{~cm}^{-1}$ for tubulin (Kristofferson et al., 1986). All polymerization reactions were performed in BRB80 as the basic buffer, with additions as described. Glycerol concentrations are volume per volume.

For polymerization, tubulin was first preincubated with nucleotides at $0^{\circ} \mathrm{C}$ for $20 \mathrm{~min}$. Polymerization was at $37^{\circ} \mathrm{C}$ for 30 min unless otherwise stated. For experiments where microtubule mass and nucleotide composition were determined by sedimentation, the micro- tubules were loaded onto a 2-ml cushion of $60 \%$ glycerol in BRB80 and sedimented $70000 \mathrm{rpm}$ in a TLA100.3 rotor (Beckman, Fullerton, CA) for $30 \mathrm{~min}$ at $35^{\circ} \mathrm{C}$ to quantitatively pellet the microtubules. The cushion was rinsed and aspirated, and the microtubules were resuspended in cold BRB80. After incubation at $0^{\circ} \mathrm{C}$ for $30 \mathrm{~min}$, the tubulin was sedimented at $40000 \mathrm{rpm}$ for $15 \mathrm{~min}$ to remove cold-stable aggregates (which were $<5 \%$ of total in all experiments), and tubulin and nucleotide concentration were measured in the cold supernatent. For experiments where microtubules were assayed rapidly after sedimentation, we used a $160-\mu l$ glycerol cushion in a TLA100 rotor (Beckman) and sedimented at $100000 \times 8$ for $5 \mathrm{~min}$.

\section{Microtubule Polymerization Rate}

These rates were obtained by direct analysis using dark-field microscopy. Detailed methods are published (Drechsel et al., 1992). Briefly, tubulin together with GMPCPP was polymerized from Rhodaminelabeled EGS-stabilized microtubule seeds (Koshland et al., 1988) that had been adsorbed to the surface of a perfusion chamber. Microtubule growth was followed on a standard microscope (Zeiss, Thornwood, NY) equipped for dark-field, with water-jacketed objective and condenser to maintain the temperature at $35^{\circ} \mathrm{C}$. Images were recorded using an SIT camera, and microtubule lengths were digitized as described (Drechsel et al., 1992).

\section{Microtubule Depolymerization Rate}

Rhodamine-labeled tubulin was polymerized at $40 \mu \mathrm{M}$ in the presence of $1 \mathrm{mM}$ GMPCPP for $10 \mathrm{~min}$. These rhodamine seeds were diluted 1000 -fold into $1.5 \mu \mathrm{M}$ tubulin, $1 \mathrm{mM}$ GMPCPP at a ratio of $1: 10$ rhodamine:unlabeled tubulin, and growth proceeded from the microtubule ends for $1 \mathrm{~h}$. These polarity-marked microtubules were then diluted $10^{6}$-fold to a final tubulin concentration 1.5 pM in BRB80 at $37^{\circ} \mathrm{C}$. At various time points, $1 \mathrm{ml}$ of the diluted mixture was removed, and glutaraldehyde was added to $0.1 \%$. After $15 \mathrm{~min}$, the fixed microtubules were sedimented onto coverslips, and the lengths of the longer of the two dim segments elongated off each bright seed, assumed to be the plus end, were digitized as described (Hyman and Mitchison, 1990).

\section{GTP Hydrolysis}

Hydrolysis of $\gamma^{32} \mathrm{P}$-GMPCPP was measured by the phosphomolybdate method after perchloric acid denaturation, modified as described (Carlier, 1989), or by TLC as above. Quantitative analysis of radioactivity on the TLC plate was performed using a phophorescence imaging machine (Molecular Dynamics, Sunnyvale, CA).

\section{RESULTS}

\section{GMPCPP Promotes Microtubule Nucleation and Polymerization}

To analyze the effect of GMPCPP on tubulin polymerization, we preincubated GMPCPP with rhodamine-labeled tubulin at $0^{\circ} \mathrm{C}$ and then warmed the mixture to $37^{\circ} \mathrm{C}$. At various time points, aliquots stabilized by dilution into buffer containing $33 \%$ glycerol and were examined by fluorescent microscopy. As previously reported, GMPCPP supported abundant nucleation and growth of microtubules (Sandoval et al., 1977; Sandoval and Weber, 1980). Figure 1 shows typical fields of microtubules polymerized from the same concentration of tubulin with GTP (a and c) and GMPCPP (b and d). The greater rate of spontaneous nucleation with GMPCPP was evident from the increased number concentration and shorter average 

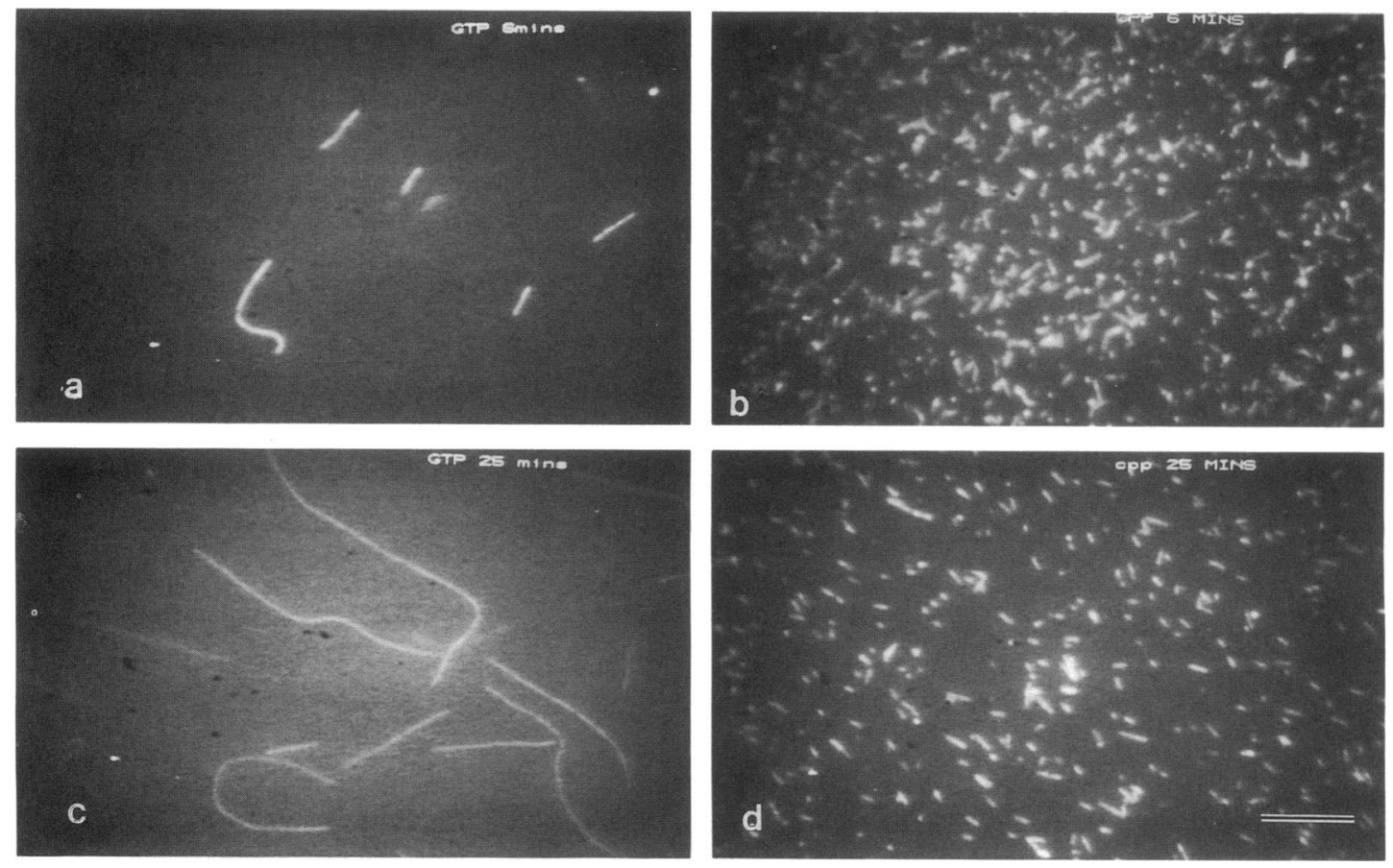

Figure 1. GMPCPP promotes polymerization and greatly enhances the nucleation of microtubules. Fifty micromolar tubulin labeled with tetramethylrhodamine to a stochiometry of 0.2 was polymerized with $1 \mathrm{mM} \mathrm{GMPCPP}$ (a and c) or $1 \mathrm{mM} \mathrm{GTP}(\mathrm{b}$ and d) and then diluted into BRB80 + glycerol for observation by fluorescence microscopy. ( $a$ and b) Six minutes of polymerization; (c and d) 26 min polymerization.

length of these microtubules (Figure $1, b$ and $d$ ). We next investigated the structure of microtubules by electron microscopy. Microtubules were polymerized with GMPCPP or GTP and either directly slam frozen for cryoelectron microscopy (Figure 2 , a and b) or absorbed to grids and negatively stained (Figure 2, dg). Again, GMPCPP microtubules were shorter and more numerous, but we saw no obvious difference between the lattice-structure of microtubules polymerized with GMPCPP or GTP. Both were ordered tubes of the reported diameter in which protofilaments with a long-pitch supertwist could be discerned. We saw no sheets or other aberrant structures formed with either nucleotide. We did notice that the GMPCPP microtubules very frequently had flush ends (arrows), whereas the GTP microtubules mostly had frayed ends. Frayed ends in GTP microtubules have been documented previously and related to their dynamic behavior (Simon and Salmon, 1990; Mandelkow and Mandelkow, 1991).

\section{Polymerization Rate With GMPCPP}

To measure the polymerization rate of GMPCPP-tubulin, we imaged microtubules growing off seeds by dark-field microscopy. We determined the elongation rate of microtubule plus-ends at various tubulin concentrations. Because GMPCPP is highly active in inducing nucleation, we could only obtain linear elongation graphs at tubulin concentrations below $1.2 \mu \mathrm{M}$. In contrast, GTP-supported polymerization cannot be measured below $2 \mu \mathrm{M}$ tubulin (Drechsel et al., 1992). Figure 3 shows a graph of polymerization rate versus tubulin concentration for microtubule plus-ends in the presence of this analogue. The polymerization onrate derived from this graph was 0.19 $\mu \mathrm{m} \cdot \mu \mathrm{M}^{-1} \cdot \mathrm{min}^{-1}$, or $5 \times 10^{6} \mathrm{M}^{-1} \mathrm{~s}^{-1}$. This value is very similar to that measured for GTP-supported polymerization using the same assay $\left(3.4 \times 10^{6} \mathrm{M}^{-1} \mathrm{~s}^{-1}\right)$ (Drechsel et al., 1992) and also to our previous estimate for GTP-supported polymerization using a fixed time-point assay $\left(3.8 \times 10^{6} \mathrm{M}^{-1} \mathrm{~s}^{-1}\right)$ (Mitchison and Kirschner, 1984) and to values obtained in other laboratories (Walker et al., 1988; Simon et al., 1992). Thus, GMPCPP is a near perfect mimic of GTP in terms of its effect on tubulin polymerization rate. By extrapolating the polymerization rate graph to zero growth-rate, we could in principle obtain the GMPCPP-tubulin critical concentration and to zero

Figure 2. GMPCPP-microtubules are structurally similar to GTP microtubules. Twenty-five micromolar tubulin together with $1 \mathrm{mM}$ GMPCPP (a and $c$ ) or $1 \mathrm{mM} \mathrm{GTP}(\mathrm{b}, \mathrm{d}-\mathrm{g}$ ) was polymerized in BRB80. Samples were either slam frozen in supercooled ethane and visualized in vitreous ice $(a$ and $b$ ) or added to electron microscope grids and negative stained with $1 \%$ uranyl acetate $(c-g)$. Note the structural similarity of microtubules polymerized with each nucleotide. The GMPCPP microtubules are much shorter and more numerous due to rapid nucleation. GMPCPP polymerized microtubules tend to have flush ends (black arrowheads), whereas GTP polymerized microtubules tend to have frayed ends (white arrowheads). 

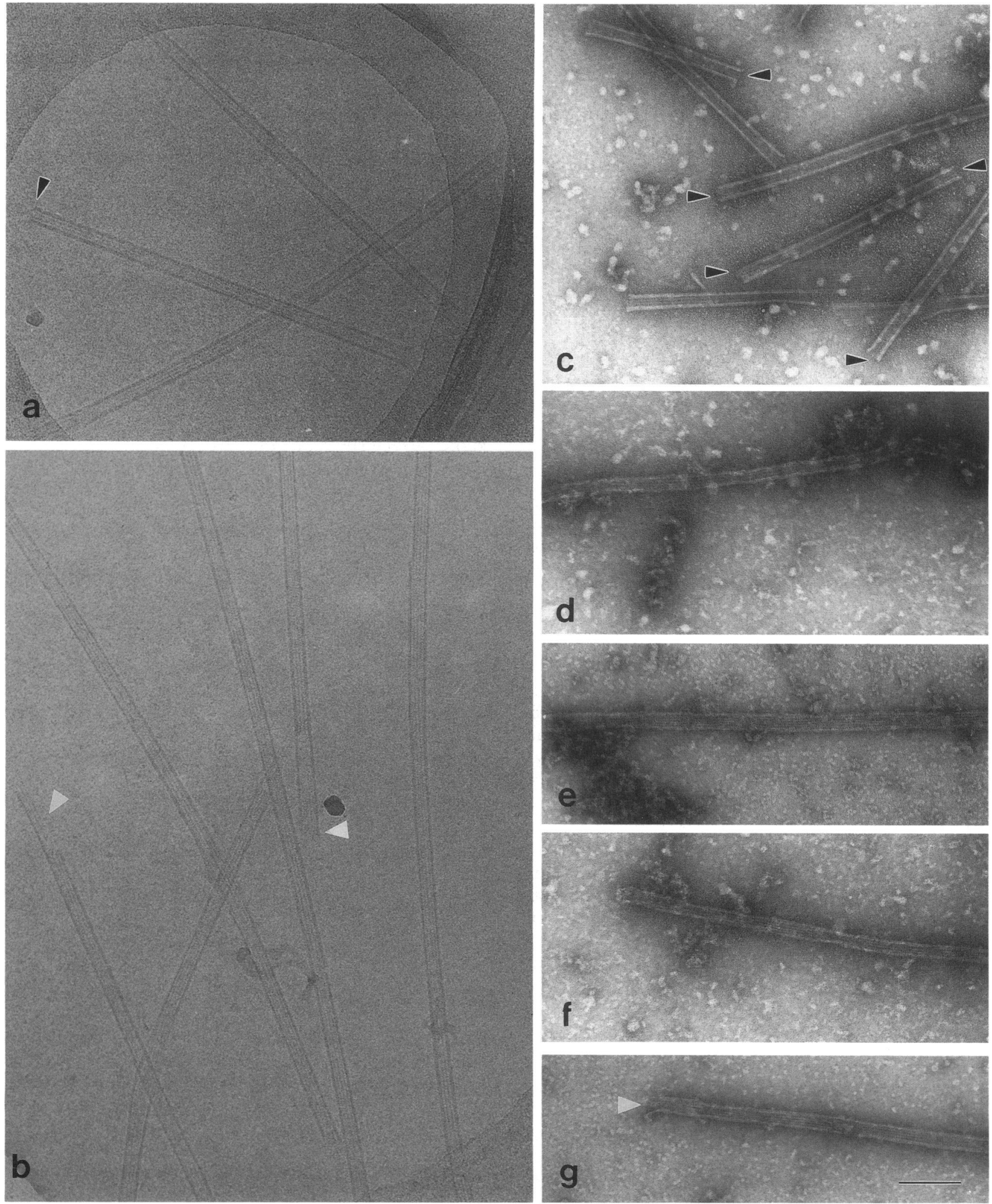


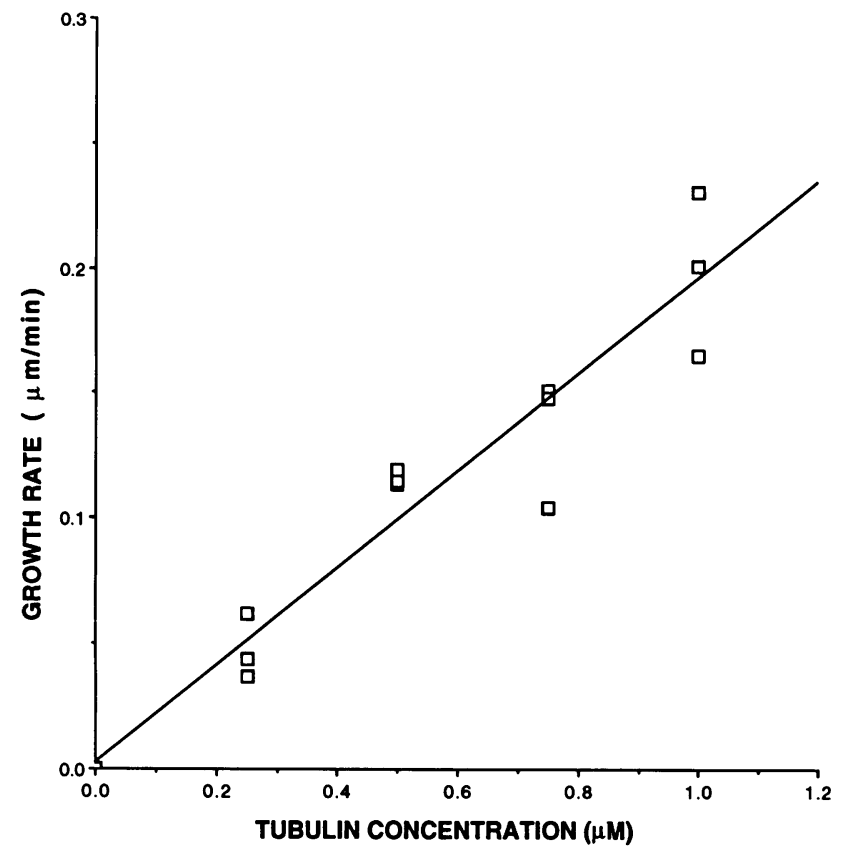

Figure 3. GMPCPP-tubulin polymerization rate. The growth rate of microtubule plus-ends elongating from stable seeds attached to a microscope side was measured as function of tubulin concentration in the presence of $1 \mathrm{mM} \mathrm{GMPCPP}$ using dark-field microscopy. Each point on the graph represents the average growth rate for 10 microtubule plus-ends in a single microscope field observed for $\geq 10 \mu \mathrm{m}$ of growth at high tubulin concentration or $20 \mathrm{~min}$ at low concentration. The line shows a least-squares fit through all the points. The slope of this line gives the average on-rate for polymerization, 0.19 $\mu \mathrm{m} \cdot \mu \mathrm{M}^{-1} \cdot \min ^{-1}$, corresponding to $5 \times 10^{6} \mathrm{M}^{-1} \mathrm{~s}^{-1}$.

tubulin the depolymerization rate. However, the best fit line gave values for both parameters not significantly different from zero using this assay, and thus they must be obtained by a different method.

\section{GMPCPP Microtubules are Relatively Stable to Dilution}

To measure the stability of GMPCPP-microtubules under conditions relevant to dynamic instability, we subjected them to isothermal dilution. Depolymerization induced by dilution is thought to proceed by the same mechanism as that occurring during the shrinking phase of dynamic instability (Walker et al., 1991). In contrast, cold and calcium induce depolymerization in a nonphysiological way, though the actual depolymerization mechanism may be related (Mandelkow and Mandelkow, 1991). To measure depolymerization rate, we polymerized highly rhodamine-labeled tubulin in the presence of GMPCPP. The resulting microtubules were then diluted into $1.5 \mu \mathrm{M}$ tubulin that was labeled to a low stochiometry with rhodamine. By operating at such a low tubulin concentration, we ensured that the dimly labeled tubulin polymerized only from the ends of the brightly labeled microtubules. This technique allows us to identify the plus-end of fixed microtubules (Hyman, 1991) and thus to measure depolymerization from a single end. The polarity marked GMPCPP-microtubules were diluted $10^{\circ}$-fold in $\mathrm{BRB} 80$ at $37^{\circ} \mathrm{C}$ to a final tubulin concentration of $1.5 \mathrm{pM}$. At successive time points, aliquots of the mixture were fixed in glutaraldehyde and centrifuged onto coverslips. The average plus-end to seed distance is plotted as a function of time in Figure 4. A least-squares fit gives a depolymerization rate of $0.23 \mu \mathrm{m} / \mathrm{h}$ or $0.10 \mathrm{~s}^{-1}$. In control experiments, microtubules were polymerized under the same conditions but with GTP in place of GMPCPP. After dilution, GTPmicrotubules disappeared completely within $60 \mathrm{~s}$ as expected. Observation of GMPCPP-microtubules using the real-time dark-field assay after flushing the chamber with buffer also gave a depolymerization rate of $\sim 0.1$ $\mathrm{s}^{-1}$ (not shown), but we do not consider the real-time analysis very reliable over such long time periods due to temperature and focus fluctuations. The GMPCPPmicrotubule depolymerization rate of $0.1 \mathrm{~s}^{-1}$ can be compared with reported off-rates for GDP subunits from plus-ends of GTP-polymerized microtubules of 300$800 \mathrm{~s}^{-1}$ under similar conditions (Mitchison and Kirschner, 1984; Walker et al., 1988, 1991; Drechsel et al., 1992; Simon et al., 1992). Therefore, the depolymeriza-

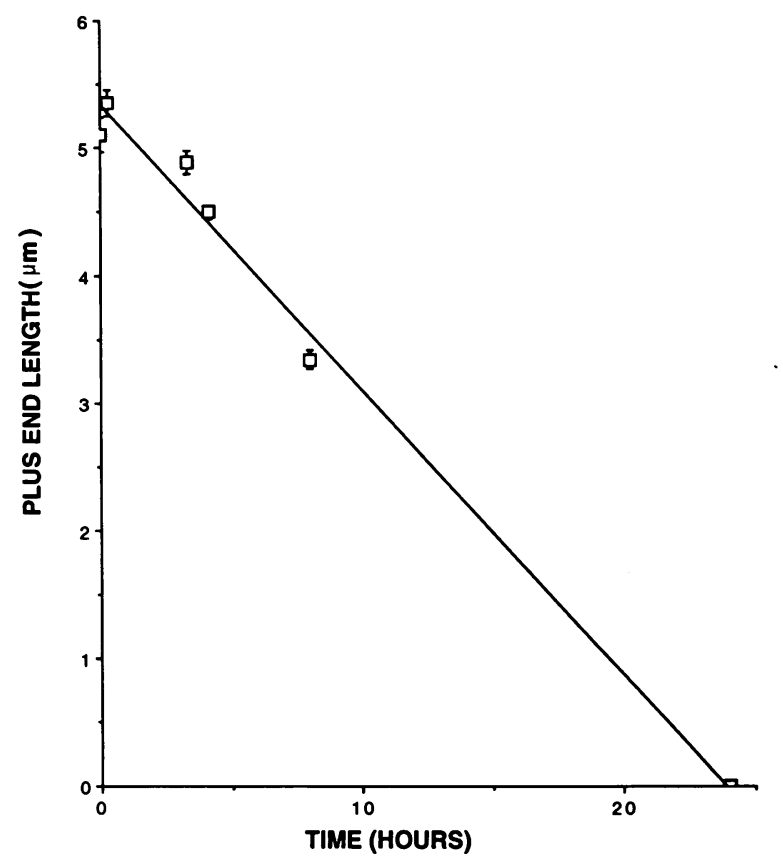

Figure 4. Dilution-induced depolymerization rate of GMPCPP microtubules. Polarity-marked GMPCPP microtubules were polymerized at $1.5 \mu \mathrm{M}$ tubulin and then diluted $10^{6}$-fold into $\mathrm{BRB80}$ at $37^{\circ} \mathrm{C}$. At various time points, aliquots were fixed with glutaraldehyde and sedimented onto coverslips. The length of the remaining microtubule segment between the plus-end and the seed was measured for $\geq 100$ microtubules, and the average plus-end segment length is plotted against time. The error bars are the $\mathrm{SE}$, and the line shows a leastsquares fit. Its slope gives an average depolymerization rate of 0.23 $\mu \mathrm{m} / \mathrm{h}$, corresponding to $0.1 \mathrm{~s}^{-1}$ from the plus end. 
tion rate of GMPCPP-microtubule is $\sim 2 \times 10^{-4}$ times that of GDP-microtubules.

Given their normal polymerization rate and very slow depolymerization rate, the critical concentration of GMPCPP microtubules is very low. We can estimate it as $0.1 / 5 \times 10^{6} \mathrm{M}$ or $20 \mathrm{nM}$. We did not try to measure this low critical concentration directly because the slow depolymerization rate would make it difficult for GMPCPP-microtubule to depolymerize to equilibrium, and attempts to measure the critical concentration by polymerization at low tubulin concentration are foiled by even the small proportion of inactive subunits that are present in even freshly cycled tubulin preparations. However, the fact that we saw appreciable polymerization onto seeds at tubulin concentrations as low as $0.3 \mu \mathrm{M}$ (Figure 3) supports the existence of a very low critical concentration.

\section{GMPCPP-Microtubules do not Show Dynamic Instability}

To determine directly whether GMPCPP inhibits dynamic instability, the growth of microtubules was followed by dark-field microscopy. Tubulin at various concentrations was polymerized off stable seeds in the presence of GMPCPP. No catastrophes (transitions from growing to shrinking) were observed as low as $0.25 \mu \mathrm{M}$ tubulin, the limit for growth detection with the assay. At this concentration of GTP-tubulin, the catastrophe rate is so high that polymerization cannot be detected at the resolution level of the light microscope (Drechsel et al., 1992). Given the slow depolymerization rate after dilution, we would not have been able to detect transitions to very slow shrinking. Nevertheless, it is clear that GMPCPP-microtubule do not show dynamic instability.

\section{Hydrolysis of GMPCPP}

To examine the hydrolysis of GMPCPP during microtubule polymerization, we synthesized a $\gamma^{32} \mathrm{P}$ derivative of the nucleotide. Tubulin $70 \mu \mathrm{M}, \gamma^{32} \mathrm{P}-\mathrm{GMPCPP}$, and unlabeled GMPCPP (final nucleotide concentration 140 $\mu \mathrm{M})$ were mixed on ice in BRB80 and then warmed to $37^{\circ} \mathrm{C}$ to polymerize. The extent of hydrolysis was determined by measuring the release of ${ }^{32} \mathrm{P}$ with time using a phosphomolybdate assay, and tubulin polymerization was checked by sedimentation. The sedimentation assay showed that after $100 \mathrm{~min}, 44 \%$ of the tubulin was in microtubules, which is lower than normal because tubulin was used straight off the photocellulose (PC) column in this particular assay and not subjected first to a polymerization-depolymerization cycle. The extent of hydrolysis per mole of tubulin polymerized is shown in Table 1. We found that after $100 \mathrm{~min}$, the amount of hydrolysis corresponded to $<0.02 \mathrm{~mol} / \mathrm{mol}$ tubulin polymer. Thus, GMPCPP is not hydrolyzed immediately after polymerization. In control experiments, we found using the same assay that alkaline phosphatase at 2 units/ml caused complete hydrolysis of $140 \mu \mathrm{M} \gamma^{32} \mathrm{P}$ GMPCPP in $1 \mathrm{~min}$ and that tubulin promoted hydrolysis of $\gamma^{32} \mathrm{P}-\mathrm{GTP}$ under polymerization conditions.

Because the data on the depolymerization rate of GMPCPP microtubules (Figure 4) required taking time points several hours after dilution, we also needed to analyze hydrolysis at long times after polymerization so that we could see to what extent the off-rate of GMPCPP-microtubules could be accounted for by hydrolysis of the bound GMPCPP. Tubulin was polymerized in the presence of $\gamma^{32} \mathrm{P}-\mathrm{GMPCPP}$ as above. The microtubules were then sedimented through a glycerol cushion and resuspended in BRB80 at $37^{\circ} \mathrm{C}$. This procedure removed free nucleotide as judged by resedimentation. When this procedure was performed using $\gamma^{32} \mathrm{P}-\mathrm{GTP}$, no radioactivity above background was recovered in the microtubule pellet, indicating normal hydrolysis of this nucleotide and release of inorganic phosphate as expected. To measure hydrolysis in the $\gamma^{32}$ P-GMPCPP-microtubules, we removed aliquots from the suspension at various time points, denatured the tubulin by addition to formamide to $67 \%$, and froze the aliquots. As a control, we took aliquots from a parallel incubation containing $\gamma^{32} \mathrm{P}-\mathrm{GMPCPP}$ but no tubulin. At the end of the incubation, we subjected all the samples to TLC analysis to measure hydrolysis. Figure 5 shows an autoradiograph of part of the chromatogram and a graphical analysis of hydrolysis. We found that $\gamma^{32} \mathrm{P}$-GMPCPP in solution underwent no detectable hydrolysis in $51 \mathrm{~h}$, beyond the $3 \%$ contamination with inorganic phosphate than was present at time zero in this preparation. In contrast, $\gamma^{32} \mathrm{P}-\mathrm{GMPCPP}$ in microtubules underwent $10 \%$ hydrolysis in the same time. Phosphate production was linear with time, and the apparent first-order hydrolysis rate was $4.3 \times 10^{-7} \mathrm{~s}^{-1}$. This rate is very slow and could perhaps be due to some contaminating nuclease. However, we found that $\gamma^{32} \mathrm{P}$ -

\begin{tabular}{cc} 
Table 1. GMPCPP hydrolysis during microtubule polymerization \\
\hline $\begin{array}{c}\text { Time } \\
(\mathrm{min})\end{array}$ & $\begin{array}{c}\text { GMPCPP hydrolysis } \\
(\mathrm{mol} \mathrm{Pi} / \mathrm{mol} \text { tubulin) }\end{array}$ \\
\hline 0 & $-0.007 \pm 0.010$ \\
1 & $0.003 \pm 0.012$ \\
10 & $0.005 \pm 0.011$ \\
100 & $0.020 \pm 0.010$ \\
\hline
\end{tabular}

Seventy micromolar tubulin and $140 \mu \mathrm{M} \gamma^{32} \mathrm{P}$-GMPCPP were polymerized in BRB80, and the amount of free phosphate was determined as a function of time using a phophomolybdate assay. The amount nucleotide hydrolyzed by tubulin was determined by counting the amount of radioactivity recovered as ${ }^{32} \mathrm{Pi}$ and subtracting the amount of ${ }^{32} \mathrm{Pi}$ present in a control reaction incubated without tubulin $(1.5 \%$ of total counts at all time points). The nucleotide hydrolyzed was then normalized to the amount of tubulin polymerized at $100 \mathrm{~min}$ (quantitated by sedimentation), which was $31 \mu \mathrm{M}(44 \%)$ in this experiment. The E-site occupancy of the microtubules pelleted at $100 \mathrm{~min}$ was $0.78 \mathrm{~mol} \mathrm{GMPCPP} / \mathrm{mol}$ tubulin in this experiment. 


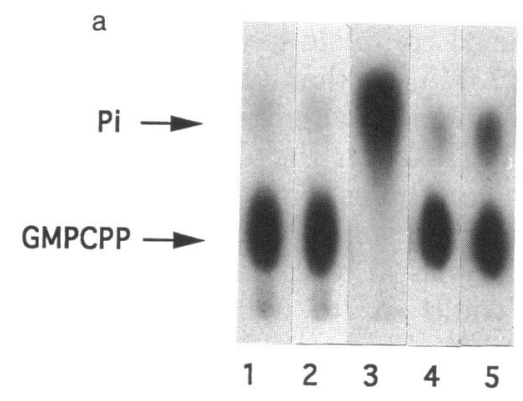

b

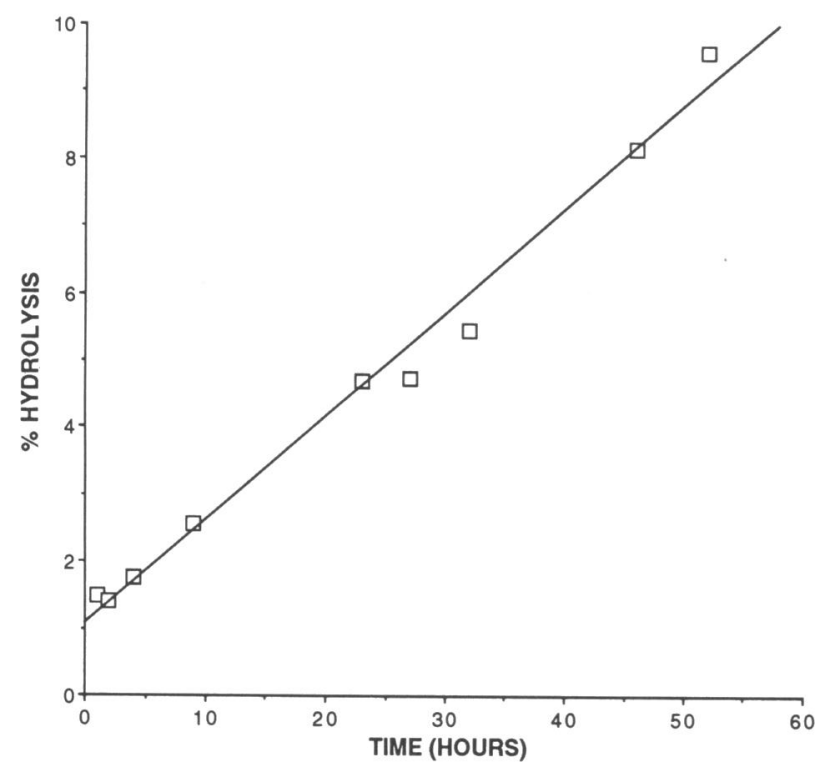

Figure 5. Hydrolysis of GMPCPP in the microtubule lattice. Microtubules were polymerized with $2 \mathrm{mM} \gamma^{32} \mathrm{P}-\mathrm{GMPCPP}$ for $30 \mathrm{~min}$ and sedimented through a 2-ml glycerol cushion to remove free nucleotide. They were resuspended in $\mathrm{BRB} 80$ and incubated at $37^{\circ} \mathrm{C}$. Under these conditions, the fraction of E-sites occupied with GMPCPP was 0.8 . Aliquots were removed, denatured by addition of formamide, and the nucleotide analyzed by TLC. Control nucleotide was incubated at $37^{\circ} \mathrm{C}$ without tubulin and then processed in the same way. (a) Portions of the autoradiograph of the chromatogram. Lane 1, nucleotide alone after $2 \mathrm{~h}$. Lane 2, nucleotide alone after $51 \mathrm{~h}$. Lane 3, nucleotide plus alkaline phosphatase, 1 unit $/ \mathrm{ml}$, after $2 \mathrm{~h}$. Lane 4 , nucleotide in microtubules after $2 \mathrm{~h}$. Lane 5 , nucleotide in microtubules after $51 \mathrm{~h}$. (b) A plot of percent hydrolysis in the microtubule sample as a function of time. The line is a linear least-squares fit, and it corresponds to a hydrolysis rate of $4.3 \times 10^{-7} \mathrm{~s}^{-1}$. The nucleotidealone sample contained $3 \%$ free phosphate, and this level did not change over $51 \mathrm{~h}$.

GMPCPP incorporated into the microtubule lattice was resistant to the action of added alkaline phosphatase under conditions where free nucleotide was rapidly hydrolyzed (not shown). Thus, we think that the slow hydrolysis rate of GMPCPP is a real consequence of being present in E-sites in the microtubule lattice. Given this very slow hydrolysis rate, we conclude that shortening of microtubules at $0.1 \mathrm{~s}^{-1}$ after dilution is due to depolymerization of GMPCPP-tubulin from the lattice rather than hydrolysis followed by depolymerization.
One prediction from its extremely slow hydrolysis is that GMPCPP should support multiple round of microtubule assembly in the absence of added nucleotide. To test this, we subjected tubulin to multiple cycles of assembly and disassembly in the presence of GMPCPP or GTP in only the first cycle. We found that GMPCPP did support multiple rounds of assembly, whereas GTP did not unless it was added freshly during each polymerization cycle (Table 2). We conclude that artificial depolymerization of GMPCPP-microtubules by cold and calcium releases tubulin subunits with unchanged GMPCPP at their E-site that are competent to repolymerize without added nucleotide.

\section{Lattice Effects on Hydrolysis Rates}

We were interested in determining whether the slow hydrolysis rate of GMPCPP was influenced by the state of the microtubule lattice, in particular by the identity of the E-site nucleotides in surrounding tubulin molecules. To measure this, we polymerized one aliquot of tubulin with $\gamma^{32}$ P-GMPCPP plus cold GMPCPP and one with $\gamma^{32}$ P-GMPCPP plus cold GTP under otherwise identical conditions. This results in one lattice where most of the E-sites ( $80 \%$ in this experiment) contain GMPCPP and a second where most sites contain GDP; a few $(\sim 1 \%)$ contain labeled GMPCPP. The microtubules were isolated by sedimentation, resuspended in a buffer containing saturating amounts of taxol to stabilize the microtubules, and hydrolysis was followed by the TLC assay. The results, shown in Figure 6, indicate that hydrolysis of $\gamma^{32} \mathrm{P}$-GMPCPP occurred at 4.8 $\times 10^{-7} \mathrm{~s}^{-1}$ in the GMPCPP lattice and at $1.5 \times 10^{-6} \mathrm{~s}^{-1}$ in the GTP lattice. Comparing GMPCPP lattice value with the value obtained above, we conclude that taxol has as negligible effect on hydrolysis rate. However, the hydrolysis rate was increased three-fold in the GDP lattice. This result increases our confidence that the slow hydrolysis rate is indeed due to the action of the intrinsic E-site GTPase mechanism and indicates that some cooperativity may exist in the hydrolysis mechanism.

Table 2. GMPCPP-tubulin can undergo multiple rounds of polymerization and depolymerization

\begin{tabular}{lrrr}
\hline & \multicolumn{3}{c}{$\begin{array}{c}\text { Microtubule yield } \\
\text { per cycle }\end{array}$} \\
\cline { 2 - 4 } & 1 & 2 & 3 \\
\hline GTP added every cycle & 75 & 53 & 48 \\
GTP added first cycle only & 75 & 0 & 0 \\
GMPCPP added every cycle & 75 & 73 & 40 \\
GMPCPP added first cycle only & 75 & 61 & 15 \\
\hline
\end{tabular}

Tubulin was subjected to rounds of polymerization, sedimentation, and depolymerization as described in MATERIALS AND METHODS, except that $\mathrm{CaCl}_{2}$ was added to $1 \mathrm{mM}$ during the cold depolymerization step and 2 mM EGTA was added before the next polymerization step. 
We were interested in the reciprocal question: whether the rate of GTP hydrolysis would be different in a GDP compared with a GMPCPP lattice. To test this, we polymerized microtubules with $\gamma^{32} \mathrm{P}-\mathrm{GTP}(<1 \mu \mathrm{M})$ and either cold GTP $(0.5 \mathrm{mM})$ or cold GMPCPP $(2.0 \mathrm{mM})$. Under these conditions, $70-80 \%$ of the E-sites are occupied by GMPCPP in the latter case (Table 3). After 5 min polymerization, the microtubules were isolated by sedimentation through a glycerol gradient. At the first time point, $10 \mathrm{~min}$ after inititating polymerization, we found that both samples contained similar amounts of $\gamma^{32} \mathrm{P}$ in the pellets, corresponding to $1-2 \%$ of the total microtubule E-sites. On TLC analysis $>95 \%$, these counts were present as inorganic phosphate in both samples. Thus, within the time resolution of our experiment $(10 \mathrm{~min})$, we were not able to detect any difference in GTP hydrolysis rate between the two lattices. However, detecting unhydrolyzed GTP in a GTP lattice requires a time resolution of better than $20 \mathrm{~s}$ (reviewed in Caplow, 1992), so this result only shows that GTP hydrolysis is not completely blocked in the GMPCPP lattice. If hydrolysis is slowed, it is probably by less than a factor of 1000 fold. Accurately measuring the effect will require developing assays with better time resolution.

\section{Stochiometry of Nucleotide Binding}

In our hydrolysis experiments, we noticed that the amount of GMPCPP incorporated into microtubules was often $<1 \mathrm{~mol} / \mathrm{mol}$ tubulin. To assay the fraction of tubulin E-sites in microtubules occupied by nucleotide added during polymerization, tubulin was preincubated with $\gamma^{32} \mathrm{P}$-GMPCPP or ${ }^{3} \mathrm{H}$-GTP at various specific activities for $20 \mathrm{~min}$ at $0^{\circ} \mathrm{C}$ in buffer with or without glycerol and then polymerized. We were able to use the $\gamma-$ labeled analogue to determine GMPCPP concentration in microtubules because of its resistance to hydrolysis demonstrated above. As shown in Table 3, the E-site occupancy in microtubules varied with nucleotide concentration during polymerization with either GMPCPP or GTP. Using $2 \mathrm{mM}$ nucleotide in glycerol buffer, the E-site occupancy reached 1.0 for GTP. This is the amount expected from the standard model for tubulin polymerization and is in agreement with previous work (Weisenberg et al., 1976; Carlier and Pantaloni, 1981). The highest E-site occupancy with GMPCPP was 0.85 , using $2 \mathrm{mM}$ nucleotide in the absence of glycerol. At lower nucleotide concentrations, the E-site occupancy was well below 1.0 with either nucleotide, showing that not every tubulin molecule that polymerizes needs to have nucleotide triphosphate bound at its E-site. We have not determined what nucleotide occupies those Esites that do not bind triphosphate, but this is presumably the GDP present from the previous polymerization (see DISCUSSION). The low E-site occupancy at low GMPCPP concentrations resulted in an amount of tubulin polymerized that was greater than the amount of nucleotide added. With $20 \mu \mathrm{M}$ GMPCPP added to 50 $\mu \mathrm{M}$ tubulin, $34.5 \mu \mathrm{M}$ tubulin was polymerized.

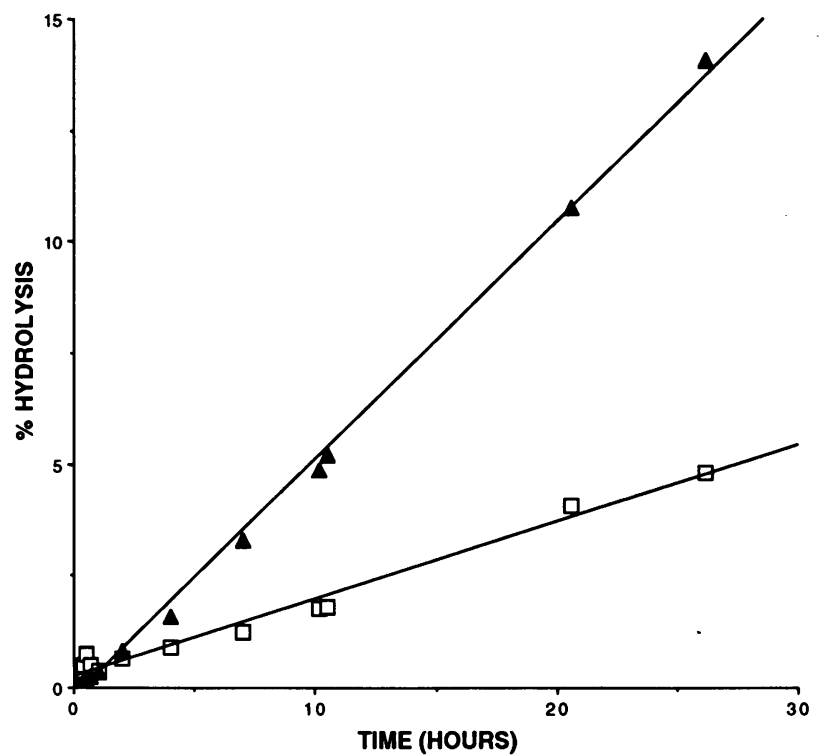

Figure 6. Hydrolysis of $\gamma^{32} \mathrm{P}-\mathrm{GMPCPP}$ in GMPCPP and GDP microtubules. Tubulin was preincubated with either $2 \mathrm{mM} \gamma^{32} \mathrm{P}$ -

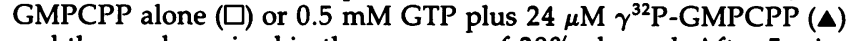
and then polymerized in the presence of $20 \%$ glycerol. After $5 \mathrm{~min}$, they were sedimented through a 200- $\mu \mathrm{l}$ glycerol cushion and then resuspended in BRB80 + $100 \mu \mathrm{M}$ taxol $+1 \%$ DMSO. The total tubulin concentration as microtubules was $\sim 50 \mu \mathrm{M}$, and the recovery was $80 \%$ for both samples. In the GMPCPP-alone polymerization, the fraction of E-sites occupied by GMPCPP was 0.8. In the GTP + GMPCPP polymerization, the fraction of E-sites occupied by GMPCPP was 0.01 . The microtubules were incubated in parallel at $37^{\circ} \mathrm{C}$, and aliquots were removed, denatured with formamide, and analyzed by TLC as in Figure 5 . The $X$-axis shows time after initiating polymerization, and the Y-axis the fraction of GMPCPP hydrolyzed. The lines are least-squares fits, and the first-order hydrolysis rates were estimated from the slope of these lines.

To determine their relative affinities for the E-site of tubulin, we investigated the competition between GMPCPP and GTP during polymerization. For comparison, we also measured competition with GMPPNP, known to have a low affinity from previous studies (Karr and Purich, 1978; O'Brien and Erickson, 1989). The results, shown in Figure 7, demonstrate that GMPCPP is a good competitor for GTP, whereas GMPPNP does not compete. The affinity of the E-site for GMPCPP is lower than that for GTP. It is difficult to determine true relative affinities of the different nucleotides from this graph because of the problem of variable site occupancy. Thus, at zero added competitor, only $22 \%$ of the E-sites of polymerized tubulin contained newly exchanged nucleotide, and this figure increased with total nucleotide concentration (see Table 3). We estimate that the apparent E-site affinity for GMPCPP is four to eightfold lower for GMPCPP than that for GTP from these data. These data make it clear why previous stripping of the E-site is required to measure GMPPNP incorporation into microtubules where competition is very poor but is not necessary with GMPCPP. 
Table 3. Quantitation of nucleotides bound to tubulin E-sites in microtubules

\begin{tabular}{lcccccc}
\hline & \multicolumn{3}{c}{ GMPCPP } & \multicolumn{3}{c}{ GTP } \\
\hline Polymerization in BRB80 & & & & & & \\
$\quad$ Nucleotide concentration $(\mu \mathrm{M})$ & 2000 & 200 & 20 & 2000 & 200 & 20 \\
Polymer yield (\%) & 79 & 83 & 69 & 51 & 61 & 19.4 \\
E-site occupancy & 0.85 & 0.48 & 0.33 & 0.69 & 0.53 & 0.27 \\
Polymerization in BRB80 $+25 \%$ glycerol & & & & & & \\
Nucleotide concentration $(\mu \mathrm{M})$ & 1850 & 300 & 50 & 1900 & 300 & 50 \\
Polymer yield (\%) & 74 & 72 & 74 & 77 & 79 & 78 \\
E-site occupancy & 0.50 & 0.39 & 0.26 & 1.03 & 0.99 & 0.80 \\
\hline
\end{tabular}

Fifty micromolar tubulin was preincubated with radiolabeled nucleotide at the indicated concentration in BRB 80 or BRB $80+25 \%$ glycerol and then polymerized. The microtubules were sedimented through glycerol cushions and depolymerized in cold BRB80. The concentration of tubulin and the nucleotide concentration were determined in the final cold supernatant. The E-site occupancy is the final radiolabeled nucleotide concentration divided by the tubulin concentration. With no added nucleotide, the polymer yield was $<0.2 \%$ under both conditions.

\section{DISCUSSION}

\section{GMPCPP as a Slowly Hydrolyzable Analogue}

Our results confirm that GMPCPP supports polymerization of normal microtubules with an increased nucleation rate and that its binding to tubulin does not require previous stripping of the endogenous E-site GDP (Sandoval et al., 1978; Sandoval and Weber, 1980). We have extended these conclusions by showing that the polymerization on-rate of GMPCPP-tubulin onto the microtubule lattice, $5 \times 10^{6} \mathrm{M}^{-1} \mathrm{~s}^{-1}$, is very similar to that of GTP-tubulin and that the binding affinity of the analog is only four to eightfold lower than that of GTP. By these criteria, we conclude that GMPCPP closely resembles GTP in the way it binds to the tubulin $\beta$ subunit and promotes the conformation necessary for polymerization. However, we find that in contrast to previous results that GMPCPP is not hydrolyzed during polymerization. Once in the microtubule lattice, it is subject to hydrolysis, but at such a slow rate $\left(0.5 \times 10^{-7}\right.$ $\mathrm{s}^{-1}$ ) as to justify calling GMPCPP an effectively nonhydrolyzable GTP analogue. Given its tight binding to the tubulin E-site and the similar polymerization rate of GTP- and GMPCPP-tubulin, we argue that GMPCPP is the most useful analogue to date for probing the role of GTP hydrolysis in microtubule polymerization. Some scientists question the relevance of results obtained from using nucleotide analogues, but most are prepared to consider results from mutant proteins. We consider the - $\mathrm{O}-$ to $-\mathrm{CH}_{2}-$ change between GTP and GMPCPP to be like a subtle mutation that preserves most of the wild-type properties while changing one key parameter, in this case hydrolysis rate.

It is difficult to say why rapid GMPCPP hydrolysis occurred during microtubule polymerization in the previously reported experiments (Sandoval et al., 1977, 1978; Sandoval and Weber, 1980) and not in the present work. Hydrolysis of GMPCPP was convincingly detected with both microtubule-associated protein (MAP)- containing and phosphocellulose-purified tubulin from rat brain. The difference in species, rat versus cow in the present study, could conceivably account for a large difference in GMPCPP hydrolysis rate by tubulin, but given the sequence conservation, this is probably less likely than methodological differences. The first Sandoval study used purified tubulin and showed that polymerization of $9 \mathrm{nmol}$ of tubulin caused hydrolysis of $50 \mathrm{nmol}$ of GMPCPP to GMPCP in $30 \mathrm{~min}$ (Sandoval et al., 1977). In a subsequent study, a time course of both polymerization and GMPCPP hydrolysis was determined in the presence of MAPs. GMPCPP hydrolysis was essentially linear with time, most hydrolysis occurring after polymerization reached a plateau (Sandoval and Weber, 1980). Because in both previous studies the final amount of GMPCPP hydrolyzed was much $>1 \mathrm{~mol} / \mathrm{mol}$ tubulin, we think it most likely that a contaminating phosphatase catalyzed the hydrolysis. In the microtubule-protein study, the authors also analyzed the nucleotide in GMPCPP-polymerized microtubule pellets and found it to be mostly GMPCP. This is more difficult to explain from the action of a contaminating phosphatase, because we found that GMPCPP incorporated into microtubules is resistant to added alkaline phosphatase. Perhaps some MAP that was present in the Sandoval assays accelerated the inherent rate of GMPCPP hydrolysis by tubulin.

\section{Role of GTP Hydrolysis in Microtubule Dynamics}

The most important result from this study is that microtubules containing bound GMPCPP depolymerize extremely slowly after isothermal dilution, with an off-rate of $0.1 \mathrm{~s}^{-1}$ compared with $\sim 500 \mathrm{~s}^{-1}$ for GDPmicrotubules. Dynamic instability is also blocked, presumably as a consequence of the block to depolymerization. Given this block and the quantitative similarity of GMPCPP to GTP in promoting polymerization, we conclude that the role of GTP hydrolysis during 
Figure 7. GMPCPP competes with GTP for the tubulin $\mathrm{E}$ site during polymerization. Fifty micromolar tubulin and $25 \mu \mathrm{M}{ }^{3} \mathrm{H}$-GTP were preincubated with varying concentrations of GTP, GMPCPP, and GMPPNP for $20 \mathrm{~min}$ at $0^{\circ} \mathrm{C}$ and then polymerized with $25 \%$ glycerol. The microtubules were sedimented through glycerol cushions, and the amount of ${ }^{3} \mathrm{H}-\mathrm{GTP}$ in the microtubule pellet was analyzed. The yield of microtubules was the same in all cases, $88 \pm 7 \%$ in this experiment. Because of the variation of site occupancy with nucleotide concentration, it is not straightforward to calculate the relative affinities of GMPCPP and GTP. At $25 \mu \mathrm{M}$ tubulin and $1 \mathrm{mM}$ GMPCPP, the E-site contains $\sim 0.08 \mathrm{~mol} / \mathrm{mol} \mathrm{GTP}$ and $0.5 \mathrm{~mol} / \mathrm{mol}$ GMPCPP. Because a 40 -fold excess of GMPCPP produces a 6-fold excess in polymer, we estimate the affinity of GMPCPP is between one-fourth and one-eighth that of GTP.

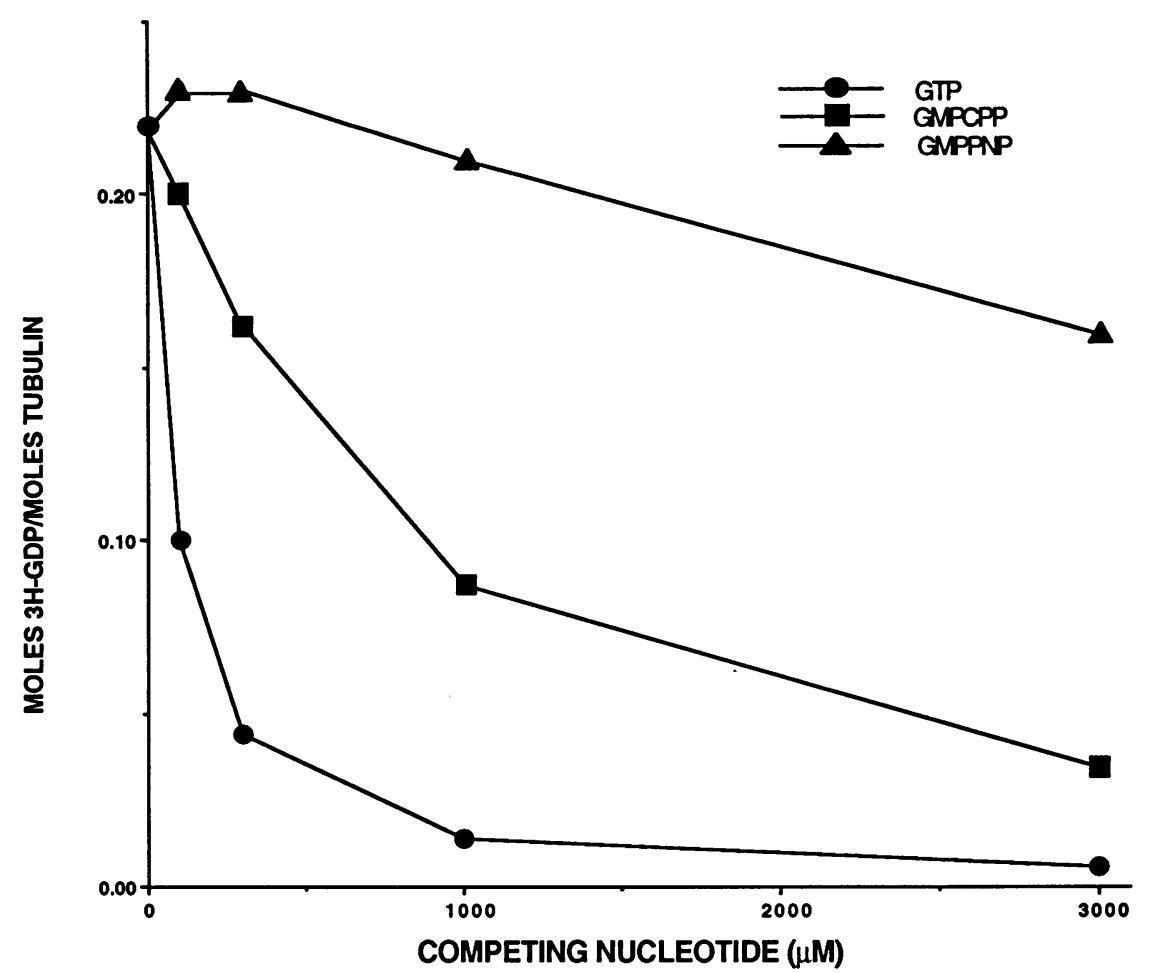

tion to tubulin E-sites in microtubules implies that not every tubulin molecule need have a triphosphate ligand to polymerize. Polymerization with substochiometric nucleotide has been observed previously (Penningroth and Kirschner, 1978), and a site-occupancy of $\sim 0.5 \mathrm{~mol}$ GDP incorporated per mole tubulin polymerized is considered standard in respected laboratories (see Stewart et al., 1990, and references therein). Presumably E-sites not occupied by GDP from hydrolysis of the GTP incorporated during the last round of polymerization are instead occupied by GDP from previous rounds. Although we did not test this directly, Hamel et al. (1986) measured directly that GDP could be incorporated into microtubules during polymerization to an extent of up to $0.3 \mathrm{~mol} /$ mol tubulin. Why some E-sites are reluctant to exchange with free nucleotide remains to be determined. One possible interpretation of heterogeneous exchange kinetics and polymerization with substochiometric GTP is that the adding unit in polymerization can be an oligomer of tubulin subunits and that only some tubulin molecules in the oligomer need have triphosphate bound to their E-site. Tubulin polymerization is thus "loosely coupled," in the terminology of Oosawa (personal communication), in the sense that there is no obligate stochiometry of coupling between polymerization and hydrolysis. This implies that the free energy per subunit invested by the polymerization process could vary with the polymerization condition, which would in turn influence the amount of pushing force that could potentially be derived from microtubule polymerization. 


\section{Control of GTP Hydrolysis}

GMPCPP has a normal phosphoanhydride bond in the $\beta-\gamma$ position, so it's extremely slow hydrolysis rate is presumably a consequence of incorrect geometry in binding to the tubulin E-site. The smaller dihedral angle of the $\mathrm{P}-\mathrm{CH}_{2}-\mathrm{P}$ bond compared with the $\mathrm{P}-\mathrm{O}-\mathrm{P}$ bond (Yount, 1975) could account for this. Because of this abnormal binding, we must be cautious in interpreting our observation that GMPCPP hydrolysis is accelerated three-fold in a microtubule where most E-sites contain GDP compared with a GMPCPP lattice. However, this observation definitely shows that the conformation of the microtubule lattice has some effect on the conformation of individual E-sites and can influence hydrolysis rate. More speculatively, it implies some cooperativity in GTP hydrolysis by microtubules. Cooperativity in GTP hydrolysis is one prediction of "vectorial" models for GTP hydrolysis by microtubules (Carlier, 1989). However, we should emphasize that the threefold rate enhancement of GMPCPP hydrolysis in a GDP lattice is a very small effect compared with enormous stimulation of GTP hydrolysis that accompanies polymerization. Furthermore, we found that GTP hydrolysis is not blocked in a GMPCPP lattice. Therefore, hydrolysis rate is primarily controlled by whether a subunit is in the lattice or not, and if the nucleotide state of surrounding subunits has any regulatory role, it is modest by comparison.

\section{GTP Hydrolysis and Nucleation}

In this study, GMPCPP greatly stimulated the rate of microtubule nucleation, confirming previous work (Sandoval et al., 1977; Sandoval and Weber, 1980). One possible explanation for this phenomenon is that during normal GTP-supported polymerization, many nucleation centers are formed transiently, but most of these fall apart due to GTP hydrolysis and the resulting destabilization of intersubunit bonds. Currently, we have little idea as to what structure constitutes the minimal nucleus for spontaneous polymerization nor do we know what constitutes the minimal tubulin oligomer that will start to hydrolyze GTP bound to E-sites. We hypothesize that GMPCPP stabilizes nucleation complexes by its resistance to hydrolysis, although the alternative possibility that GMPCPP promotes nucleation by strengthening some unusual bonding interactions cannot be ruled out. Inside the cell, spontaneous nucleation is generally undesirable because it would tend to randomize the spatial organization of microtubules. We propose that in addition to promoting dynamic instability, GTP hydrolysis by tubulin has the important biological role of suppressing spontaneous nucleation of microtubules. Extrapolating from this, the nucleating material of the centrosome could function in part by suppressing GTP hydrolysis. Recently, $\gamma$-tubulin has been implicated directly in nucleation by centrosomes (Joshi et al., 1992; Stearns and Kirschner, personal com- munication). Is $\gamma$-tubulin perhaps deficient in GTP hydrolysis or in its ability to trigger hydrolysis in a $\beta$ subunit that it contacts?

\section{Technical Uses of GMPCPP}

One reason for writing this article was to popularize GMPCPP as a tool in microtubule research. We have found it to be very useful for making stable microtubule segments without taxol, for example, as part of polaritymarked microtubules for motor-protein assays (Hyman, 1991). The extremely low critical concentration for microtubule polymerization with GMPCPP, combined with the ability to dissolve these microtubules in the cold and then simply exchange the GMPCPP for GTP, may make the analogue useful for purifying tubulin from difficult sources. Finally, the fact that $\gamma^{32} \mathrm{P}$ GMPCPP can be used to probe the hydrolytic activity of the tubulin E-site in microtubules may make it useful for identifying cellular factors that either speed up or slow down this activity. Such experiments are much more difficult with $\gamma^{32}$ P-GTP because the inherent hydrolysis rate is so fast.

\section{ACKNOWLEDGMENTS}

We thank Marc Kirschner for donating the last batch of commercial GMPCPP, which was discovered during a freezer clean-out, and for many helpful discussions. We also thank Linda Amos, Tim O'Brien, Joe Howard, and Ted Salmon for stimulating discussion. This work was supported by NIH grant GM-29565, as well as fellowships from the Searle and Packard foundations to T.J.M. A.A.H. is a Lucille P. Markey scholar, and this work was funded in part by a grant from the Lucille P. Markey charitable trust.

\section{REFERENCES}

Arai, T., and Kaziro, Y. (1976). Effect of guanine nucleotide on the assembly of brain microtubules: ability of guanylyl imidodiphosphate to replace GTP in promoting the polymerization of microtubules in vitro. Biochem. Biophys. Res. Commun. 69, 369-376.

Caplow, M. (1992). Microtubule dynamics. Curr. Opin. Cell Biol. 4, 58-65.

Carlier, M.F. (1989). Role of nucleotide hydrolysis in the dynamics of actin filaments and microtubules. Int. Rev. Cytol. 115, 139-170.

Carlier, M.F., and Pantaloni, D. (1981). Kinetic analysis of guanosine $5^{\prime}$-triphosphate hydrolysis associated with tubulin polymerization. Biochemistry 20, 1918-1924.

Chen, Y.-D., and Hill, T.-L. (1985). Monte Carlo study of the GTP cap in a five-start helix model of a microtubule. Proc. Natl. Acad. Sci. USA 82, 1131-1135.

DeBrabander, M., Geuens, G., Nuydens, R., Willebrords, R., and DeMey, J. (1981). Microtubule assembly in living cells after release form nocodazole block. The effect of metabolic inhibitors, taxol and pH. Cell Biol. Int. Rep. 5, 913-920.

Drechsel, D.N., Hyman, A.A., Cobb, M.H., and Kirschner, M.W. (1992). Modulation of the dynamic instability of tubulin assembly by the microtubule-associated protein tau. Mol. Biol. Cell 3, 1141-1154.

Gelfand, V.I., and Bershadsky, A.D. (1991). Microtubule dynamics: mechanism, regulation and function. Annu. Rev. Cell Biol. 7, 93-116. 
Hamel, E.H., Batra, J.K., and Lin, C.M. (1986). Direct incorporation of guanosine-5'-diphosphate into microtubules without guanosine5'-triphosphate hydrolysis. Biochemistry 25, 7054-7062.

Hyman, A.A. (1991). Preparation of marked microtubules for the assay of the polarity of microtubule-based motors by fluoescence. J. Cell Sci. Suppl. 14, 125-127.

Hyman, A.A., Drexel, D., Kellog, D., Salser, S., Sawin, K., Steffen, P., Wordeman, L., and Mitchison, T.J. (1991). Preparation of modified tubulins. Methods Enzymol. 196, 478-485.

Hyman, A.A., and Mitchison, T.J. (1990). Modulation of microtubule stability by kinetochores in vitro. J. Cell Biol. 110, 1607-1616.

Joshi, H.C., Monica, J.P., McNamara, L., and Cleveland, D.W. (1992). Gamma-tubulin is a centrosomal protein required for cell cycle-dependent microtubule nucleation. Nature 356, 80-83.

Karr, T.L., and Purich, D.L. (1978). Examination of tubulin-nucleotide interactions by protein fluorescence quenching measurements. Biochem. Biophys. Res. Commun. 84, 957-961.

Kirschner, M.W. (1978). Microtubule assembly and nucleation. Int. Rev. Cytol. 54, 1-71.

Kirschner, M.W., and Mitchison, T.J. (1986). Beyond self assembly: from microtubules to morphogenesis. Cell 45, 329-342.

Koshland, D., Mitchison, T.J., and Kirschner, M.W. (1988). Chromosome movement driven by microtubule depolymerization in vitro. Nature 311, 499-504.

Kristofferson, D., Mitchison, T., and Kirschner, M.W. (1986). Direct observation of steady-state microtubule dynamics. J. Cell Biol. 102, 1007-1019.

Mandelkow, E.M., and Mandelkow, E. (1991). Microtubule dynamics and microtubule caps: a time resolved cryo-electron microscopy study. J. Cell Biol. 114, 977-992.

Mejillano, M.R., Barton, J.S., Nath, J.P., and Himes, R.H. (1990). GTP analogues interact with the tubulin exchangeable site during assembly and upon binding. Biochemistry 29, 1208-1216.

Mitchison, T.J., and Kirschner, M.W. (1984). Dynamic instability of microtubule growth. 312, 237-242.

Myers, T.C., Nakamura, K., and Danielzadeh, A.B. (1965). Phosphonic acid analogs of nucleotide phosphates. III. The synthesis of adenosine5 '-methylenediphosphonate, a phosphonic acid analog of adenosine $5^{\prime}$ diphosphate. J. Org. Chem. 30, 1517-1520.

O'Brien, E.T., and Erickson, H.P. (1989). Assembly of pure tubulin in the absence of free GTP: effect of magnesium, glycerol, ATP, and nonhydrolyzable GTP analogs. Biochemistry 28, 1413-1422.

Penningroth, S.M., and Kirschner, M.W. (1977). Nucleotide binding and phosphorylation in microtubule assembly in vitro. J. Mol. Biol. 115, 643-673.

Penningroth, S.M., and Kirschner, M.W. (1978). Nucleotide specificity in microtubule assembly in vitro. Biochemistry 17, 734.
Purich, D.L., and Kristoffersen, D. (1984). Microtubule assembly: a review of progress, principles, and perspectives. Adv. Protein Chem. $36,133-212$

Sandoval, I.V., Jameson, J.L., Niedel, J., MacDonald, E., and Cuatrecasas, P. (1978). Role of nucleotides in tubulin polymerization: effect of guanosine 5'-methylene diphosphonate. Proc. Natl. Acad. Sci. USA $75,3178-3182$

Sandoval, I., MacDonald, E., Jameson, J.L., and Cuatrecasas, P. (1977) Role of nucleotide in tubulin polymerization: effect of guanylyl $5^{\prime}$ methylenediphosphonate. Proc. Natl. Acad. Sci. USA 74, 4881-4885.

Sandoval, I.V., and Weber, K. (1980). Guanosine 5'(a,b-methylene)triphosphate enhances specifically microtubule nucleation and stops the treadmill of tubulin protomers. J. Biol. Chem. 255, 69666974.

Seckler, R., Wu, G.M., and Timasheff, S.N. (1990). "Interactions of tubulin with guanylyl-(beta-gamma-methylene)diphosphonate. Formation and assembly of a stochiometric complex." J. Biol. Chem. 265, 7655-7661.

Simon, J.R., Parsons, S.F., and Salmon, E.D. (1992). Buffer conditions and non-tubulin factors critically affect the microtubule dynamic instability of sea urchin egg tubulin. Cell Motil. Cytoskeleton 21, 1-14.

Simon, J.R., and Salmon, E.D. (1990). The structure of microtubule ends during the elongation and shortening phases of dynamic instability examined by negative stain electron microscopy. J. Cell Sci. 96, 571-582.

Stewart, R.J., Farrell, K.W., and Wilson, L. (1990). Role of GTP hydrolysis in microtubule assembly: evidence for a coupled hydrolysis mechanism. Biochemistry 29, 6489-6498.

Walker, R.A., O'Brien, E.T., Pryer, N.K., Sobeiro, M.F., Voter, W.A., Erickson, H.P., and Salmon, E.D. (1988). Dynamic instability of individual microtubules analysed by video light microscopy: rate constants and transition frequencies. J. Cell Biol. 107, 1437.

Walker, R.A., Pryer, N.K., and Salmon, E.D. (1991). Dilution of individual microtubules observed in real time in vitro: evidence that the cap size is small and independent of elongation rate. J. Cell Biol. $114,73-82$.

Wehland, J., and Sandoval, I. (1983). Cells injected with guanosine 5 '-[a,b,-methylene] triphosphate, and a-b nonhydrolysable analog of GTP, show anomalous patterns of tubulin polymerization affecting cell translocation, intracellular movement, and the organization of Golgi elements. Proc. Natl. Acad. Sci. USA 80, 1938-1941.

Weisenberg, R.C., and Deery, W.J. (1976). Role of nucleotide hydrolysis in microtubule assembly. Nature $263,792-793$.

Weisenberg, R.C., Deery, W.J., and Dickinson, P.J. (1976). Tubulinnucleotide interactions during polymerization and depolymerization of microtubules. Biochemistry 15, 4248-4254.

Yount, R.G. (1975). ATP analogs. Adv. Enzymol. Relat. Areas Mol. Biol. 43, 1-56. 